\title{
Relation between brain displacement and local cerebral blood flow in patients with chronic subdural haematoma
}

\author{
S Inao, T Kawai, R Kabeya, T Sugimoto, M Yamamoto, N Hata, T Isobe, J Yoshida
}

\begin{abstract}
Objectives-To evaluate the relation between brain displacement, clinical signs and symptoms, and local cerebral blood flow (1CBF) in patients with chronic subdural haematoma (CSDH).

Methods-Forty five patients (age range 58-87 years, mean 71.9 (SD 8.4)) with unilateral CSDH were studied. Patients were categorised into three groups: $I$, headache $(n=16)$; II, paresis $(n=14)$; and III, mental change $(n=15)$. T1 weighted MR images were obtained in all patients preoperatively. Quantitative values of maximum haematoma thickness, midline shift, and brain rotation angle were measured on axial and coronal MR images. In 21 patients, 1CBF was measured by $\mathrm{Xe}$ enhanced CT. Values for $1 \mathrm{CBF}$ were obtained in selected regions of interest in the frontal cortex, thalamus, and hemisphere on both the haematoma and contralateral sides.
\end{abstract}

Results-The ICBF reduction in the ipsilateral frontal cortex showed the best linear correlation with haematoma thickness $(r=0.57)$, whereas the reduction in the ipsilateral thalamus had the most significant correlation with pineal shift $(r=0.65)$ and third ventricle incline $(r=0.67)$. In patients with paresis, 1CBF decreased significantly on the ipsilateral side of both the frontal cortex and thalamus $(p<0.05)$, whereas patients with mental change showed a significant reduction of $1 \mathrm{CBF}$ on both sides of the thalamus $(p<0.01)$ and in the ipsilateral frontal cortex $(p<0.01)$. Conclusions-The 1CBF reduction and clinical symptoms correlated well with local brain displacement in patients with CSDH. The ICBF in the central cerebral area including the thalamus was reduced in patients with clinical signs. The mental changes found were thought to derive from mild impairment of consciousness due to upper brain stem displacement. (F Neurol Neurosurg Psychiatry 2001;71:741-746)

Keywords: brain displacement; cerebral blood flow; chronic subdural haematoma; hemiparesis

Correspondence to: Dr S Inao, Department of Neurosurgery, Japanese Red Cross, Nagoya First Hospital, 3-35 Michishita, Nakamura, Nagoya 453-8511, Japan

sinao@nagoya-1st.jrc.or.jp

Received 19 October 1999 and in final form

19 April 2001

Accepted 20 May 2001 The exact mechanism by which brain function is impaired when compressed and displaced by an intracranial mass is unclear. Several authors have analyzed the relation between the clinical symptoms and the brain shift or transtentorial herniation in patients with supratentorial masses. ${ }^{1-3}$ However, these reports showed only a relation between brain displacement or transtentorial herniation and consciousness disturbance.

There have been only a few reports regarding local cerebral blood flow $(1 \mathrm{CBF})$ and metabolism in patients with chronic supratentorial masses. ${ }^{4-8}$ Although they showed reduction of $\mathrm{CBF}$ and metabolism in patients with clinical manifestations, the contribution of brain displacements to these clinical manifestations was not elucidated in detail.

In our previous study, ${ }^{9}$ we showed the relations between brain displacement on MRI and electrophysiological changes measured by brain stem auditory evoked response (BAER) in patients with chronic subdural haematoma $(\mathrm{CSDH})$. The results indicated that the latency of central conduction time was prolonged and brain stem kinking occurred when the supratentorial mass became larger. In the present study, we evaluated the relation between brain displacement, clinical signs and symptoms, and $1 \mathrm{CBF}$ reduction in patients with CSDH. We also discuss the mechanism by which an intracranial mass may cause brain dysfunction.

\section{Materials and methods}

We studied 45 patients (age range $58-87$ years; mean 71.9 (SD 8.4)) with unilateral CSDH. Informed consent was obtained from all patients or their families. Patients were grouped into three by the symptoms and signs as follows; headache alone without any neurological deficits (group I: headache, $n=16$ ), paresis of the limbs without mental changes (group II: paresis, $\mathrm{n}=14$ ), and mental change with or without paresis (group III: mental change, $n=15$ ). In group III, 12 patients were hemiparetic. The average age was 72.9 in group I, 70.9 in group II, and 71.9 in group III. Mental changes in patients of group III included consciousness disturbance (delirium, drowsiness, and fluctuating consciousness, which ranged from 9 to 13 on the Glasgow coma scale) and dementia (impairment of memory and intellect with intact sensorium). A history of trauma in the preceding months was elicited in $76 \%(34 / 45)$ of the patients. None of the patients had bilateral lesions or other cerebral pathology. All but two patients in group I were operated on for the evacuation of the haematoma soon after the examinations.

Both coronal and axial T1 weighted MR 

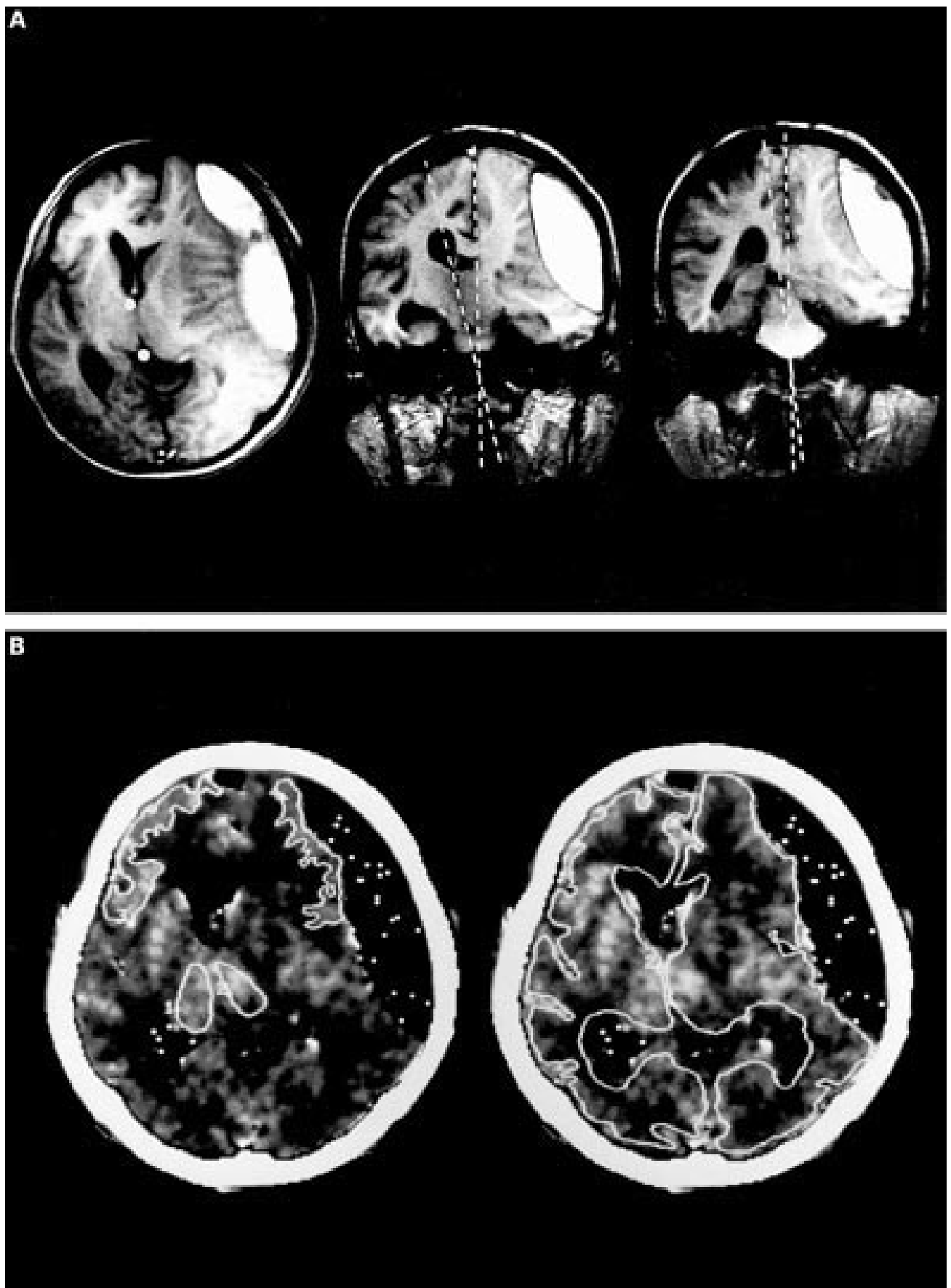

Figure 1 (A) Maximum haematoma thickness and brain rotation angles (the incline of the third ventricle and the axis of brain stem) on coronal images, and midline shifts of the septum pellucidum and pineal body on axial images were measured. (B) The grey scale images of a CBF map created by Xe enhanced CT showing the placement of regions of interest for the frontal cortex - thalamus (left) and whole cerebral hemisphere (right).

(the incline of the third ventricle and the axis of brain stem) were obtained on the coronal images (fig $1 \mathrm{~A}$ ). The midline shifts of the septum pellucidum and pineal body were measured on the axial images. The values were converted to true dimensions using the linear scale on the film images. Details were described in our previous report. ${ }^{9}$

In 21 patients, including seven in group I, eight in group II, and six in group III, $1 \mathrm{CBF}$ was measured preoperatively with $\mathrm{Xe}$ enhanced $\mathrm{CT}$. In all the patients having $\mathrm{CBF}$ measurements, mean arterial blood pressure (MABP) and arterial blood gas were also examined, and found to be within a physiological range (MABP 80-120 mm Hg; $\mathrm{PaCO}_{2}$, 33-48 mm $\mathrm{Hg}$; pH, 7.35-7.45). An Xe-CT CBF study was carried out with a TCT-900S CT scanner (Toshiba Corp, Tokyo, Japan), and an AZ-723 Xe inhalation system with end tidal Xe monitor (Anzai Sogyo Co, Tokyo, Japan). All patients inhaled a mixture of $30 \%$ Xe in oxygen through a face mask for 6 minutes. Brain CT images were obtained every 45 seconds for 6 minutes during the wash in period and for another 6 minutes during the wash out period. A CBF mapping image was created by processing the endotidal build up curve and tissue build up 


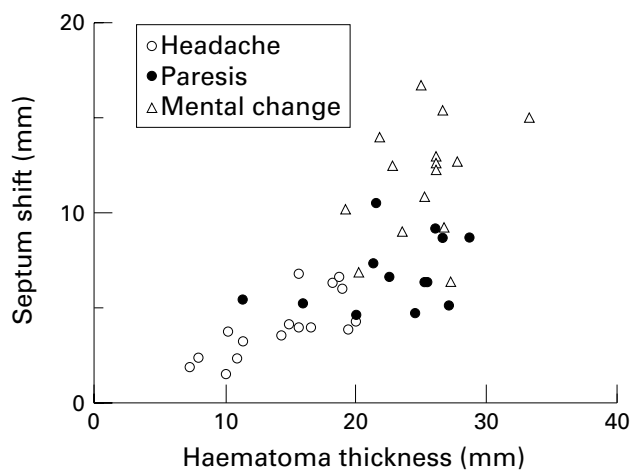

Figure 2 Relations among the haematoma thickness, septum shift, and clinical symptoms in the three groups were plotted. In group I (headache); haematoma thickness was $<20 \mathrm{~mm}$ and septum shift was $<10 \mathrm{~mm}$ in all cases. All but three patients in group II (paresis) had a haematoma thickness $>20 \mathrm{~mm}$ and septum shift $<10 \mathrm{~mm}$. All but one patient in group III (mental change) showed a haematoma thickness $>20 \mathrm{~mm}$, and all but four showed a septum shift $>10 \mathrm{~mm}$.

curve using 02C-CBF imaging software (Toshiba Corp). The scan consisted of a single slice in a plane through the pineal body parallel to the orbitomeatal line at the level of the basal ganglia in all but four patients. In each patient, absolute values of $1 \mathrm{CBF}$ were obtained in selected regions of interest in the frontal cortex, thalamus, and hemisphere on both the haematoma and contralateral sides (fig $1 \mathrm{~B}$ ). Details were described in our previous papers. ${ }^{1011}$ The flow value of the thalamus in four patients was not obtained because the CBF image was scanned in a more superior slice due to the location of the haematoma. Normal $1 \mathrm{CBF}$ values were obtained in five volunteers (all men; age range 53-65, mean 57.8 years), and mean values were calculated in 10 hemispheres: $37.2(2.6 \mathrm{ml}) / 100 \mathrm{~g} / \mathrm{min}$ in the frontal cortex, 58.2 (SD7.2) $\mathrm{ml} / 100 \mathrm{~g} / \mathrm{min}$ in the thalamus, and $31.2(\mathrm{SD} 3.9 \mathrm{ml}) / 100 \mathrm{~g} / \mathrm{min}$ in the hemisphere.

The mean $1 \mathrm{CBF}$ value in each region was plotted against the values for maximum haematoma thickness, brain midline shift, and brain rotation angle for all subjects. Correlations between the brain displacement and $1 \mathrm{CBF}$ were analyzed by linear regression analysis. The parameters of brain displacement were compared between the groups by one factor factorial analysis of variance (ANOVA). Values for $1 \mathrm{CBF}$ of each group were also compared in each brain area by one factor factorial ANOVA.

Table 1 Maximum haematoma thickness, brain shift, and rotation

\begin{tabular}{llll}
\hline & $\begin{array}{l}\text { Group I } \\
\text { (headache) }\end{array}$ & $\begin{array}{l}\text { Group II } \\
\text { (paresis) }\end{array}$ & $\begin{array}{l}\text { Group III } \\
\text { (mental change) }\end{array}$ \\
\hline $\begin{array}{l}\text { Haematoma thickness (mm) } \\
\text { Brain shift or rotation: }\end{array}$ & $14.3(4.3)$ & $23.0(5.0)^{\star}$ & $25.4(3.5)^{\star}$ \\
$\quad$ Septum shift (mm) & $4.0(1.6)$ & $7.0(2.1)^{\star}$ & $11.6(3.0) \dagger$ \\
Pineal shift (mm) & $2.9(1.3)$ & $5.2(1.8)^{\star}$ & $7.9(1.7) \dagger$ \\
Third ventricle rotation $\left({ }^{\circ}\right)$ & $5.0(2.5)$ & $8.3(2.9)$ & $12.3(3.9) \dagger$ \\
Brain stem rotation $\left(^{\circ}\right)$ & $3.5(2.4)$ & $5.1(2.9)$ & $7.8(2.7) \dagger$ \\
\hline
\end{tabular}

Values are mean (SD).

${ }^{\star} \mathrm{p}<0.01$ compared with group I.

$t \mathrm{p}<0.01$ compared to group I and group II.

\section{Results}

RELATION BETWEEN CLINICAL SYMPTOMS AND

BRAIN DISPLACEMENT

The relations among the maximum haematoma thickness, septum shift, and clinical symptoms in the three groups are shown in figure 2. In group I (headache), haematoma thickness was less than $20 \mathrm{~mm}$ and septum shift was less than $10 \mathrm{~mm}$ in all cases. All but three patients in group II (paresis) had a haematoma thickness of more than $20 \mathrm{~mm}$ and septum shift of less than $10 \mathrm{~mm}$. All but one patient in group III (mental change) showed a haematoma thickness of more than $20 \mathrm{~mm}$, and all but four patients showed a septum shift of more than $10 \mathrm{~mm}$. The data on maximum haematoma thickness and brain displacements including septum shift, pineal shift, third ventricle rotation, and brain stem rotation in the three groups are summarised in table 1. The haematoma thickness in groups II and III was significantly greater than in group I $(p<0.01)$, but there was no significant difference between group II and group III. Both shifts of septum and pineal body in group II were greater than those in group I $(p<0.01)$, and both shifts in group III were larger than those in group II $(p<0.01)$. Neuroaxis rotations of the third ventricle and brain stem in the coronal plane were significantly greater in group III than in either group I or group II $(\mathrm{p}<0.01)$.

RELATION BETWEEN BRAIN DISPLACEMENT AND LCBF

The $1 \mathrm{CBF}$ value of each patient for each region was plotted against haematoma thickness and brain displacements. The 1CBF decreased linearly in all areas corresponding to the severity of brain displacements. The reduction of $1 \mathrm{CBF}$ in the frontal cortex on the haematoma side had the best linear correlation with haematoma thickness (fig $3 \mathrm{~A} ; \mathrm{y}=39.0-0.4 \mathrm{x}$, $r=0.57$ ), whereas the reduction of $1 \mathrm{CBF}$ in the thalamus on the haematoma side had the most significant correlation with the pineal shift (fig $3 \mathrm{~B}$; $\mathrm{y}=56.2-2.8 \mathrm{x}, r=0.65)$ and the third ventricle incline ( $r=0.67$, not shown). The magnitude of the $1 \mathrm{CBF}$ reduction, indicated as the incline of the line obtained by the linear regression analysis in fig 3, was greater in the thalamus than in the frontal cortex due to the brain displacement.

RELATION BETWEEN CLINICAL SYMPTOMS AND LCBF

Figures $3 \mathrm{~A}$ and $\mathrm{B}$ show the relation between $1 \mathrm{CBF}$ and the clinical symptoms in each patient. The patients with mental change had the greatest reduction of $1 \mathrm{CBF}$, those with paresis had a moderate reduction of $1 \mathrm{CBF}$, and those with headache alone had the least reduction of $1 \mathrm{CBF}$ in both the frontal cortex and thalamus on the haematoma side. These data are summarised in table 2 and figure 4 . In group I (headache), ICBF did not change significantly compared with the normal values in any region even though the normal values were obtained from non-age matched volunteers. In group II (paresis), lCBFs in all regions 

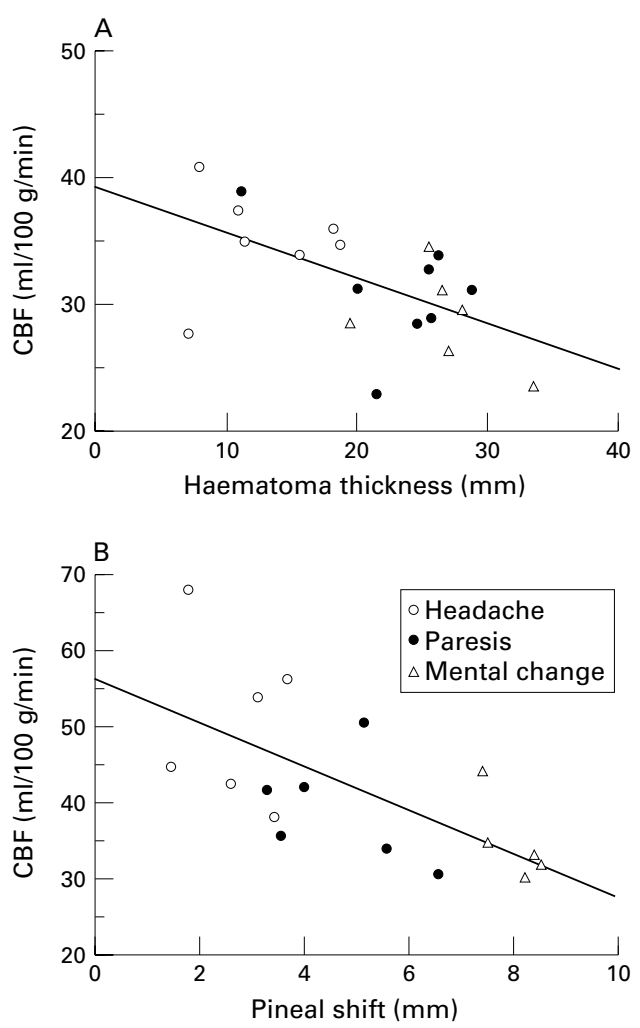

Figure 3 The $l C B F$ value of each patient was plotted against maximum hematoma thickness and brain displacements. The reduction of $l C B F$ in the frontal cortex in the haematoma side had the best linear correlation with the haematoma thickness ( $A ; y=39.0-0.4 x, r=0.57$ ), whereas the reduction of $l C B F$ of the thalamus in the haematoma side had the most significant correlation with the pineal shift $(B ; y=56.2-2.8 x, r=0.65)$ and the third ventricle incline ( $r=0.67$, not shown).

decreased significantly compared with the normal values in each region. However, only 1CBFs in the frontal cortex and thalamus on the haematoma side were significantly different from those in group I ( $p<0.05$, each). In group III (mental change), 1CBFs in all regions also showed a significant reduction compared with the normal values. In comparison, the lCBFs in group III in both the frontal cortex and thalamus on the haematoma side significantly decreased compared with group I $(\mathrm{p}<0.01)$. Furthermore, $1 \mathrm{CBF}$ in the thalamus on the contralateral side in group III significantly decreased compared with that in group II $(\mathrm{p}<0.05)$.

Table 2 Local cerebral blood flow (lCBF)

\begin{tabular}{|c|c|c|c|c|c|}
\hline & & Normal & $\begin{array}{l}\text { Group I } \\
\text { (headache) }\end{array}$ & $\begin{array}{l}\text { Group II } \\
\text { (paresis) }\end{array}$ & $\begin{array}{l}\text { Group III } \\
\text { (mental change) }\end{array}$ \\
\hline \multicolumn{6}{|c|}{$1 \mathrm{CBF}(\mathrm{ml} / 100 \mathrm{~g} / \mathrm{min}):$} \\
\hline \multirow[t]{2}{*}{ Frontal cortex } & $\mathrm{H}$ & & $35.0(3.9)$ & $31.0(4.6)^{\star}$ & $28.8(3.8) \ddagger$ \\
\hline & $\mathrm{C}$ & $37.2(2.6)$ & $34.5(5.4)$ & $32.8(5.3) \dagger$ & $30.5(4.5) \rrbracket$ \\
\hline \multirow[t]{2}{*}{ Thalamus } & $\mathrm{H}$ & & $50.8(11.1)$ & $39.3(7.1)^{\star}$ & $34.7(5.4) \ddagger$ \\
\hline & $\mathrm{C}$ & $58.2(7.2)$ & $51.4(9.5)$ & $42.9(7.5)$ & $31.1(5.7)$ \\
\hline \multirow[t]{2}{*}{ Hemisphere } & $\mathrm{H}$ & & $27.1(5.9)$ & $23.4(3.7) \oint$ & $22.4(4.5) \S$ \\
\hline & $\mathrm{C}$ & $31.2(3.9)$ & $27.4(5.9)$ & $25.0(3.8) \S$ & $23.7(4.7) \S$ \\
\hline
\end{tabular}

Values are mean (SD); normal values were obtained from five volunteers (10 hemispheres); $\mathrm{H}=$ haematoma side; $\mathrm{C}=$ contralateral side.

${ }^{\star} \mathrm{p}<0.01$ compared with normal and $\mathrm{p}<0.05$ compared with group I.

$t \mathrm{p}<0.05$ compared with normal.

$\neq \mathrm{p}<0.01$ compared with both normal and group I.

$\S \mathrm{p}<0.01$ compared with normal.

$\lceil\mathrm{p}<0.01$ compared with group 1 and $\mathrm{p}<0.05$ compared with group II.
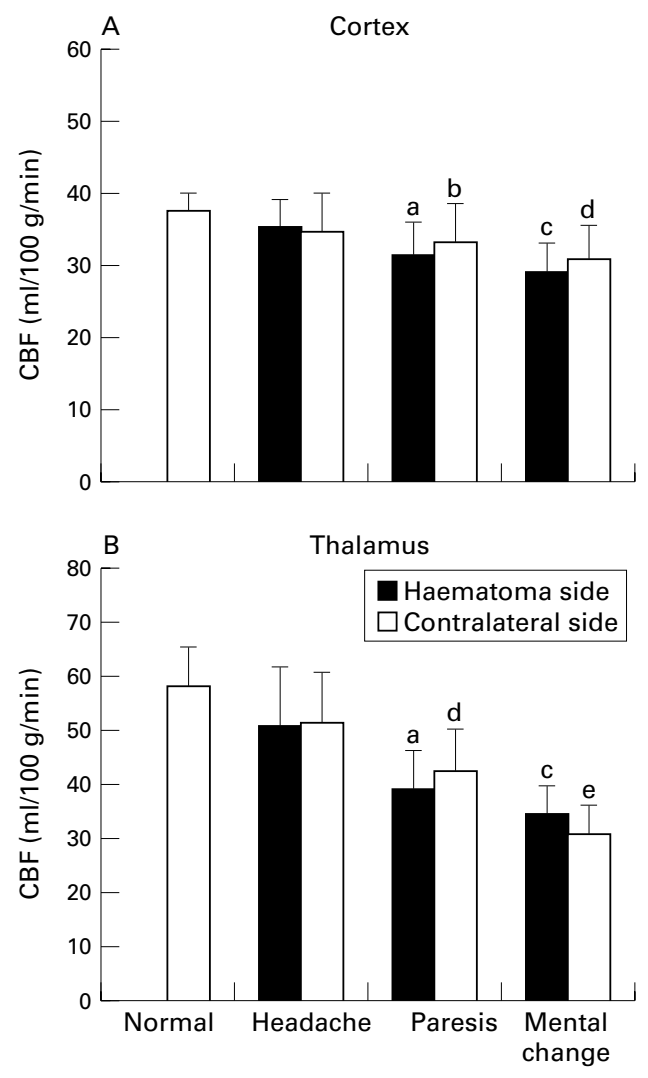

Figure 4 In group II (paresis), lCBFs of the frontal cortex and thalamus in the haematoma side were significantly different from those in group I $(p<0.05$, each). In group III (mental change), lCBFs of both the frontal cortex and thalamus in the haematoma side significantly decreased compared with group I $(p<0.01)$, and $l C B F$ of the thalamus in the contralateral side also significantly decreased compared with that in group II $(p<0.05)$. a, $p<0.01$ compared with normal values and $p<0.05$ compared with group $I ; b, p<0.05$ compared to normal values; $c, p<0.01$ compared with both normal values and group I; $d, p<0.01$ compared with normal values; $e, p<0.01$ compared with group I and $p<0.05$ compared with group

\section{Discussion}

BRAIN DISPLACEMENT AND CLINICAL SYMPTOMS Altered mental function and hemiparesis are among the most common features of CSDH in older patients. ${ }^{12}$ Mental changes have been categorised into three types ${ }^{13}$ : consciousness disturbance, dementia, and mixed type. In this study, we included all patients with mental changes in one category (group III) because of the difficulty in differentiating between consciousness disturbance and dementia due to the wide variation in intellectual change and fluctuation of consciousness level.

Chronic subdural haematoma has a relatively uniform location, and the brain displacement is primarily affected by the size of the haematoma. In our previous study, ${ }^{9}$ we demonstrated the relations among the brain displacements of the various parts of the midline structure associated with $\mathrm{CSDH}$. The data indicated that the midline structures of the brain shifted and rotated when the haematoma thickness increased beyond the level of spatial compensation, and that midbrain kinking occurred in an advanced stage. In the present study, we evaluated the relation between the brain displacement and clinical symptoms. The 
data showed that the clinical symptoms were related to both the haematoma thickness and brain displacements. In the patients without neurological deficits (group I), the septum shift was limited to about $4 \mathrm{~mm}$, although the haematoma compressed the superficial structure of the cortex to a greater degree (measured as maximum haematoma thickness; mean thickness $14 \mathrm{~mm}$ ). In such cases, the mass effect on the midline structures might be reduced by cerebral atrophy. When the haematoma thickness increased beyond about 20 $\mathrm{mm}$, both the superficial and deep brain structures were deformed, and the neurological signs of hemiparesis and mental changes occurred in relation to the degree of midline displacement.

Several authors have reported relations between disturbance of consciousness and brain displacement with unilateral masses using neuroimaging techniques. Ropper ${ }^{1}$ reported that in patients with acute masses, horizontal displacement of the brain above the tentorium measured by CT is more closely related to impairment of the conscious level than to the brain stem compression due to the tentorial herniation. From further analysis using MRI, Ropper $^{2}$ stressed that most patients with acute unilateral masses have upper brain stem displacement predominantly due to horizontal shift at or above the tentorium. Reich et al demonstrated the relation between clinical signs and brain herniation with supratentorial masses by measurements of midsagittal MRI in both acute and chronic masses. Their results showed that in acute illnesses MRI changes anticipated or confirmed clinical signs of brain herniation, whereas in chronic cases clinical and MRI findings were less correlated. Our data also showed a wide range of brain displacement in each group. Although all the previous reports focused mainly on the relation between brain displacement and disturbance of consciousness, our data indicated graded changes of clinical symptoms, including nonneurological signs, hemiparesis, or mental change, related to brain displacement with a supratentorial mass.

BRAIN DISPLACEMENT AND ICBF

The present study demonstrated that $1 \mathrm{CBF}$ in all areas decreased linearly with an increase in haematoma thickness or the severity of brain displacement. Although the $1 \mathrm{CBF}$ in the ipsilateral frontal cortex was most affected by haematoma thickness, in the ipsilateral thalamus it correlated best with the displacement of the deep brain structures, including the pineal shift and neuroaxis rotation. The cause of hemiparesis was reported to be the reduced $1 \mathrm{CBF}$ in the rolandic cortex ${ }^{5}$ or in deep structures including the thalamus. ${ }^{6}$ Our data for hemiparetic patients (group II) showed that the $1 \mathrm{CBF}$ was reduced in both the ipsilateral frontal cortex and thalamus. The displacement of the superficial structures, measured as haematoma thickness, was related to the displacement of the deep brain structures when the haematoma thickness increased beyond spatial compensation, so hemiparesis may be induced by the reduction of $1 \mathrm{CBF}$ in the ipsilateral pyramidal tract, including both the frontal cortex and subcortical structures.

Tanaka et $a l^{14}$ reported that the cause of clinical signs including hemiparesis and mental change was the $1 \mathrm{CBF}$ reduction in the deep structures, especially in the thalamus. Because patients with hemiparesis and mental change were analyzed together, distinct differences in the mechanism of hemiparesis and mental change could not be determined. Our data clearly demonstrate that the $1 \mathrm{CBF}$ reduction in the ipsilateral thalamus alone did not relate to mental changes; changes in cognition were associated with the reduction on both sides of the thalamus. This bilateral $1 \mathrm{CBF}$ reduction in the thalamus was thought to be closely related to displacement and dysfunction in the upper brain stem (midbrain). Our previous data on the relation between the brain stem displacement and the prolongation of central conduction time on BAER also support the importance of midbrain kinking at the advanced stage of brain displacement. ${ }^{9}$ These results suggest that the mental changes in patients with $\mathrm{CSDH}$ were mainly disturbance in consciousness due to the dysfunction of the deep brain structures. Although it seems possible that higher cognitive function was impaired by the decreased $1 \mathrm{CBF}$ in the ipsilateral frontal cortex, this may be ruled out in the present cases because the reduction of $1 \mathrm{CBF}$ in the ipsilateral frontal cortex also existed in the paretic patients without mental changes (group II).

Normal ICBF values in our institute were not age matched for this study. Because CBF is thought to be reduced in elderly subjects, ${ }^{15}$ the age matched normal values might be lower than the values used. Thus, there may be no significant differences in the data of $1 \mathrm{CBF}$ in clinical groups compared with normal values. However, comparisons among the clinical groups I to III showed significant differences in $1 \mathrm{CBF}$ (see results section).

MECHANISM OF CLINICAL SYMPTOMS

It is still unclear how intracranial masses impair brain function - that is, reduced metabolic demand, reduced $\mathrm{CBF}$, or raised intracranial pressure (ICP). Little is known about the metabolic status of the brain with CSDH. Ishikawa $e t a l^{8}$ reported that in $\mathrm{CSDH}$, the $1 \mathrm{CBF}$ and cerebral metabolic rate for oxygen $\left(\mathrm{CMRO}_{2}\right)$ in the affected hemisphere were reduced but the oxygen extraction fraction (OEF) was raised with normal cerebral blood volume, and that these changes were pronounced mainly in the anterior central cerebral area. Although they thought that the hypoperfusion and hypometabolism in their patients were insufficient to cause neuronal energy failure, $\mathrm{CMRO}_{2}$ as the index of metabolic status significantly decreased. Our previous finding of brain $\mathrm{pH}$ in patients with $\mathrm{CSDH}$ also showed a decrease in $\mathrm{pH}$ (acidosis), possibly due to the hypoperfusion. ${ }^{7}$ Brodersen $e t a l^{4}$ suggested that $\mathrm{CBF}$ reduction in patients with $\mathrm{CSDH}$ was probably secondary to a reduced metabolic demand. Although there are still no definite conclusions in this regard, our data indicated 
that $1 \mathrm{CBF}$ reduction and clinical symptoms were well correlated with brain displacement. Further studies are needed to determine the primary cause of clinical presentation due to the brain displacement - that is, reduced metabolic demand or reduced CBF. Increased ICP with mass lesions may cause the generalised reduction of CBF. Although ICP was not measured in our study, it is reportedly often near normal in older patients with $\mathrm{CSDH} .{ }^{16}$

\section{Conclusion}

The present study demonstrated that $1 \mathrm{CBF}$ does not always decrease in the area adjacent to a haematoma, but often does decrease in the central cerebral area including the thalamus. Reduction in CBF and clinical symptoms were well correlated with brain displacement. Mental changes found were thought to derive from mild impairment of consciousness due to upper brain stem displacement.

We thank Dr M Furuse, Dr H Kuchiwaki, Dr T Hirano, Dr K Yoshida, Mrs H Koida, and Mr A Izawa of Nakatsugawa Municipal General Hospital for their technical assistance and management of the patients.

1 Ropper AH. Lateral displacement of the brain and level of consciousness in patients with an acute hemispheral mass. consciousness in patients with an
N Engl F Med 1986;314:953-8.

2 Ropper AH. A preliminary MRI study of the geometry of brain displacement and level of consciousness with acute intracranial masses. Neurology 1989;39:622-7.

3 Reich JB, Sierra J, Camp W, et al. Magnetic resonance imaging measurements and clinical changes accompanying transtentorial and foramen magnum brain herniation. Ann Neurol 1993;33:159-70.
4 Brodersen P, Gjerris F. Regional cerebral blood flow in patients with chronic subdural hematomas. Acta Neurol patients with chronic subdur
Scandinav 1975;51:233-29.

5 Ikeda K, Ito H, Yamashita J. Relation of regional blood flow to hemiparesis in chronic subdural hematoma. Surg Neurol 1990;33:87-95

6 Tanaka A, Yoshinaga S, Kimura M. Xenon-enhanced computed tomographic measurement of cerebral blood flow in patients with chronic subdural hematomas. Neurosurgery 1990;27:554-61.

7 Yoshida K, Furuse M, Izawa A, et al. Dynamics of cerebral metabolism in patients with chronic subdural hematoma evaluated with phosphorous $31 \mathrm{MR}$ spectroscopy before and after surgery. AfNR Am f Neuroradiol 1994;15:1681-6.

8 Ishikawa T, Kawamura S, Hadeishi H, et al. Cerebral blood flow and oxygen metabolism in hemiparetic patients with chronic subdural hematoma: quantitative evaluation using positron emission tomography. Surg Neurol 1995;43:130-7.

9 Inao S, Kuchiwaki H, Kanaiwa $\mathrm{H}$, et al. Magnetic resonance imaging assessment of brain distortion associated with a supratentorial mass. F Neurol Neurosurg Psychiatry 1993;56: $280-5$.

10 Yoshida K, Furuse M, Izawa A, et al. Dynamics of cerebral blood flow and metabolism in patients with cranioplasty as evaluated by ${ }^{133} \mathrm{Xe} \mathrm{CT}$ and ${ }^{31} \mathrm{P}$ magnetic resonance spectroscopy. F Neurol Neurosurg Psychiatry 1996;61:166-171.

11 Inao S, Kuchiwaki H, Yoshida J, et al. Magnetic resonance imaging quantitation of superior sagittal sinus flow: correimaging quantitation of superior sagittal sinus fow: correcomputed tomography. Neurol Res 1997;19:35-40.

12 Fogelholm R, Heiskanen O, Waltimo O. Chronic subdural hematoma in adults. Influence of patient's age on symptoms, signs, and thickness of hematoma. 7 Neurosurg $1975 ; 42: 43-6$

13 Black D. Mental changes resulting from subdural haematoma. Br f Psychiatry 1984;145:200-3.

14 Tanaka A, Kimura M, Yoshinaga S, et al. Computed tomography and cerebral blood flow correlations of mental changes in chronic subdural hematoma. Neurosurgery 1992;30:370-8.

15 Frackowiak RSJ, Lenzi GL, Jones T, et al. Quantitative measurement of regional cerebral blood flow and oxygen metabolism in man, using ${ }^{15} \mathrm{O}$ and positron emission tomography: theory, procedure and normal values. $7 \mathrm{Com}$ put Assist Tomogr 1980;4:727-36.

16 Tanaka A, Nakayama Y, Yoshinaga S. Cerebral blood flow and intracranial pressure in chronic subdural hematomas. Surg Neurol 1997;47:346-51. 